\title{
Effect of UV Laser Radiation on "Positive Regulation of Telomere Maintenance" in Saccharomycescerevisiae
}

\author{
Babak Arjmand $^{1}$, Nahid Safari-Alighiarloo ${ }^{2}{ }^{\circledR}$, Mohhamadreza Razzaghi $^{3}{ }^{\oplus}$, Mostafa Rezaei Tavirani $^{*^{*}}$ \\ (D), Mohammad Rostami Nejad ${ }^{(\mathbb{D}}$, Majid Rezaei Tavirani ${ }^{\circledR}$, Vahid Mansouri ${ }^{\circledR}$, Reza Vafaee $^{7^{(}}$ \\ ${ }^{1}$ Cell Therapy and Regenerative Medicine Research Center, Endocrinology and Metabolism Molecular-Cellular \\ Sciences Institute, Tehran University of Medical Sciences, Tehran, Iran \\ ${ }^{2}$ Endocrine Research Center, Institute of Endocrinology and Metabolism, Iran University of Medical Sciences, \\ Tehran, Iran \\ ${ }^{3}$ Laser Application in Medical Sciences Research Center, Shahid Beheshti University of Medical Sciences, Tehran, Iran \\ ${ }^{4}$ Proteomics Research Center, Faculty of Paramedical Sciences, Shahid Beheshti University of Medical Sciences, \\ Tehran, Iran \\ ${ }^{5}$ Research Institute for Gastroenterology and Liver Diseases, Gastroenterology and Liver Diseases Research Center, \\ Shahid Beheshti University of Medical Sciences, Tehran, Iran \\ ${ }^{6}$ Faculty of Medicine, Iran University of Medical Sciences, Tehran, Iran \\ ${ }^{7}$ Critical Care Quality Improvement Research Center, Faculty of Paramedical Sciences, Shahid Beheshti University \\ of Medical Sciences, Tehran, Iran
}

*Correspondence to Mostafa Rezaei Tavirani, Proteomics Research Center, Faculty of Paramedical Sciences, Shahid Beheshti University of Medical Sciences, Tehran, Iran. Email: tavirany@yahoo.com

Received: October 17, 2021 Accepted: December 21, 202 Published online December 28 2021

\section{Introduction}

Ultraviolet (UV) ray molecular damage to skin cells may lead to various complications. UV can be divided into three types: A, B and C. The third type with a wavelength of 100 to $280 \mathrm{~nm}$ is absorbed by the Earth's atmosphere ozone. But type A with a wavelength of 430 to $400 \mathrm{~nm}$ and $10 \%$ of type B with a wavelength of 280 to $320 \mathrm{~nm}$ could reach the earth's surface. In environmental abundance, UV rays can cause various complications in the human body, such as sunburn ${ }^{1}$ and various skin cancers. ${ }^{2}$ Damage to the cornea of the eye is also one of these complications. ${ }^{3}$ The direct absorption of UV energy by cells leads to damage to DNA and proteins and indirectly to the formation of free radicals. ${ }^{4} \mathrm{UV}$ radiation with a short wavelength is carcinogenic (280-315 nm) and has been the focus of most researches. However, it should be noted that long-wavelength UV radiation can also cause cell damage. Short-wavelength UV causes damage to DNA, while long-wavelength radiation acts indirectly, and the produced free oxygen radicals from the reactions could be absorbed by other cell molecules. ${ }^{5}$ Molecular functions of UV lead to various skin carcinomas and are still unclear as it is a mutagen and has the properties of a tumor initiator and a tumor promoter. ${ }^{1}$ However, the benefits of UV by mediating the natural synthesis of vitamin D and endorphins in skin are inevitable. ${ }^{6}$ Atrophy, pigmentation

Please cite this article as follows: Arjmand B, Safari-Alighiarloo N, Razzaghi M, Rezaei Tavirani M, Rostami Nejad M, Rezaei Tavirani $\mathrm{M}$, et al. Effect of uv laser radiation on "positive regulation of telomere maintenance" in saccharomyces cerevisiae. J Lasers Med Sci. 2021;12:e87. doi:10.34172/jlms.2021.87. 
changes, wrinkling and malignancy are among the profound health risks carried by excessive exposure to UV. ${ }^{7}$ The three most common types of skin cancer; basal cell carcinoma, squamous cell carcinoma, and malignant melanoma are epidemiologically linked to UV radiation and molecular mechanism of these disorders is not clear properly. ${ }^{8}$ Such genetic factors as polymorphism of the Melanocortin 1 receptor (MCR1) gene could influence the risk of UV mediated skin disease. This gene is involved in UV sensitivity and skin cancer. ${ }^{9}$ MCR1 found on the surface of melanocytes could bind to a-melanocyte stimulating hormone (MSH) and transmit differentiation into the cell through the activation of adenylyl cyclase and cAMP production. ${ }^{10,11}$ The risk of skin cancer is influenced by UV exposure and skin pigmentation. ${ }^{12}$ Malignant melanoma of skin arising from epidermal melanocytes is a metastatic-prone malignancy. ${ }^{13}$ Two major non-melanomatous skin cancers are basal cell carcinomas and squamous cell carcinomas, both derived from keratinocytes. UV mutations in cancer-relevant genes such as p53 tumor suppressor in squamous cell carcinoma are well characterized. ${ }^{14}$ The exome analysis of melanomas has revealed that UV radiation has a direct mutagenic role in the pathogenesis of melanoma. ${ }^{15}$ UV-induced DNA mutations are causative factors of melanoma. ${ }^{16}$ Mutations caused by UV lead to ROS generation, such as superoxide anion, hydrogen peroxide and the hydroxyl radical. ${ }^{17}$ Nucleotides are susceptible to free radical injury, and the oxidation of nucleotide bases causes mutagenesis. ${ }^{18} \mathrm{UV}$ radiation induces abnormality in transcription, DNA replication, and base pairing. ${ }^{19}$ UV-induced photoproducts and other bulky DNA lesions could be repaired by nucleotide excision repair as an evolutionary mechanism. ${ }^{20}$

Saccharomyces cerevisiae is one of the simple biological models for studying the genome and how UV rays affect biological molecules. Facile genetic manipulation and the large degree of homology between genes in yeast and humans make it useful, ${ }^{21}$ and about $30 \%$ of known genes involved in human disease have yeast orthologues. ${ }^{22}$ Many yeast genes involved in damage response checkpoints and DNA repair by UV irradiation have human orthologues and their mutation induces cancer sensitivity. ${ }^{23}$

Network analysis is a useful method for screening the dysregulated genes based on properties of the studied genes in interaction with the other elements of the analyzed network. ${ }^{24}$ A network contains nodes connected to each other via edges and there are few nodes playing a critical role in network construction named central nodes. ${ }^{25}$ Analyzing the network interactions between proteins and genes modified by UV in yeast has made us develop the relationship between proteins and genes important in this regard.

In the present study, the gene expression profiles of $S$. cerevisiae cells which are treated by the UV laser were compared with the controls. Significantly expressed genes were determined to construct a network, and critical elements of the network such as hubs and bottlenecks were introduced to analyze interactions between key genes to find the prominent effects of UV on S. cerevisiae genes.

\section{Materials and Methods}

Original data were extracted from the reported document by Hauser et al. ${ }^{26}$ As it is published by this research group, the gene expression profiles of $S$. cerevisiae samples in the presence of the UV laser at 30 seconds and 15 minutes after irradiation harvesting were compared with control profiles. The full details of the methods are described in the published data by the authors. The data were screened based on $P$ value $<0.05$ and ratio change $>2$. The characterized genes were included in an interactome by the "protein query" of the STRING database via Cytoscape software. The network was analyzed by "NetworkAnalyzer" application of Cytoscape. Mean + 2SD was considered as the cut-off value of the degree to identify hub nodes. Five percent of top nodes based on betweenness centrality were determined as bottlenecks. Common hubs and bottlenecks were selected as hubbottleneck nodes. The other group of nodes (top 5\% nodes based on closeness centrality and stress) was identified. Common genes between the two central groups and the hub-bottleneck nodes were determined as critical DEGs.

The connection between the critical DEGs was formed via STRING, and a sub-network was constructed. The critical nodes were screened via action map analysis. The nodes interacted by directional connections which were corresponded to activation, inhibition, and expression actions via CluePedia application of Cytoscape. The key critical DEGs were enriched for biological terms by using ClueGO plugin of Cytoscpe.

\section{Results}

A total of 452 differentially expressed genes (DEGs) were screened among the reported genes. Since 4 proteins were not identified in data banks, 448 individuals were a candidate for more investigations. The network including 19 isolated genes, 1 paired node, and 427 interacted individuals by 3617 edges was constructed via undirected connections. Degree value 47 was determined as the cutoff value to determine the hub nodes. Based on degree value 47, 19 top nodes were identified as hubs (see Table 1). Analysis revealed that 11 DEGs among the determined hubs were hub-bottleneck nodes. More investigation indicated that all hub-bottlenecks were presented in the top nodes which were characterized based on closeness centrality and stress values. The binding property of the critical DEGs was investigated via network analysis. Connections between the hub-bottleneck nodes are illustrated in Figure 1. As it is depicted in Figure 1, HSP104 
is connected to the 10 first neighbors, while ZWF1 and GLK1 are linked to 5 critical DEGs.

The action map including activation, inhibition, and expression is shown in Figure 2. As shown in Figure 2, there is no expression relationship between the critical DEGs and 4 DEGs including RPN11, UBI4, HSP82, and HSC82 (as the key critical DEGs) that are connected by inhibition and activation links.

One class (positive regulation of telomere maintenance)

Table 1. Hub Nodes of the Network and the Related Centrality Parameters Including Degree, Betweenness Centrality (BC), Closeness Centrality (CC), and Stress

\begin{tabular}{|c|c|c|c|c|c|}
\hline No. & Display name & Degree & BC & $\mathrm{CC}$ & Stress \\
\hline 1 & HSP104 & 79 & 0.0238 & 0.4754 & 86408 \\
\hline 2 & HSP82 & 76 & 0.0335 & 0.4671 & 93394 \\
\hline 3 & HSP42 & 75 & 0.0376 & 0.4723 & 86366 \\
\hline 4 & RPN11 & 73 & 0.0437 & 0.4566 & 97224 \\
\hline 5 & HSC 82 & 72 & 0.0300 & 0.4620 & 85568 \\
\hline 6 & CDC48 & 63 & 0.0352 & 0.4465 & 76188 \\
\hline 7 & UBI4 & 61 & 0.0197 & 0.4401 & 46348 \\
\hline 8 & PRX1 & 60 & 0.0285 & 0.4595 & 71656 \\
\hline 9 & GLK1 & 60 & 0.0312 & 0.4532 & 68380 \\
\hline 10 & HSP78 & 57 & 0.0122 & 0.4503 & 42096 \\
\hline 11 & HSP26 & 55 & 0.0122 & 0.4522 & 39472 \\
\hline 12 & TPS1 & 55 & 0.0141 & 0.4508 & 41198 \\
\hline 13 & PNC1 & 53 & 0.0134 & 0.4470 & 31464 \\
\hline 14 & DCS2 & 53 & 0.0088 & 0.4320 & 26994 \\
\hline 15 & RTC3 & 51 & 0.0117 & 0.4286 & 28452 \\
\hline 16 & ZWF1 & 49 & 0.0248 & 0.4465 & 58312 \\
\hline 17 & ATG8 & 48 & 0.0280 & 0.4378 & 59254 \\
\hline 18 & RPN10 & 48 & 0.0081 & 0.4247 & 25578 \\
\hline 19 & GLC3 & 47 & 0.0111 & 0.4316 & 24950 \\
\hline
\end{tabular}

Note: The nodes of No. 1-9 and 16-17 are hub-bottleneck nodes.

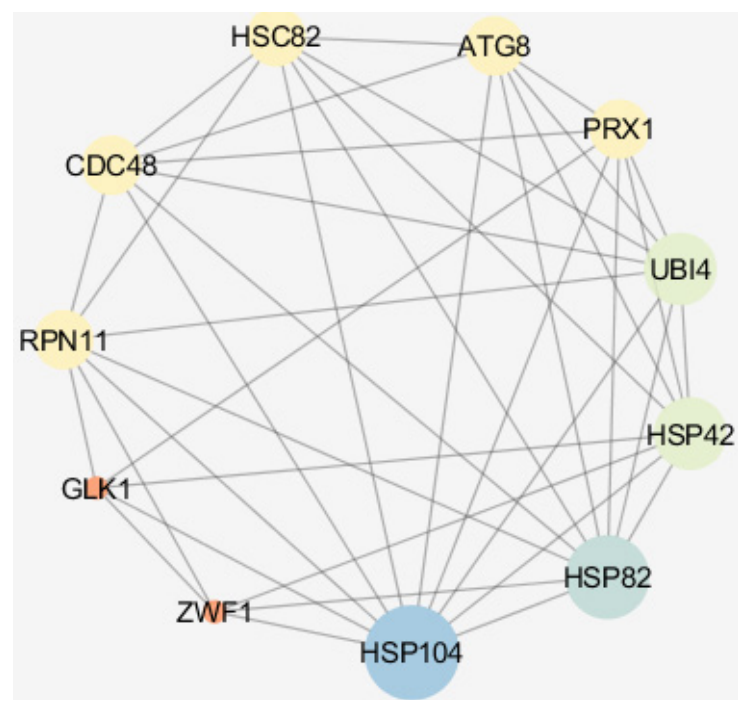

Figure 1. A Sub-network Including the Hub-Bottleneck Nodes. The bigger size and blue color refer to higher value of degree. including 4 biological terms ("positive regulation of telomere maintenance", "positive regulation of telomere maintenance via telomerase", "positive regulation of telomere maintenance via telomere lengthening", and "positive regulation of DNA biosynthetic process" are related to the key critical DEGs (see Figure 3).

\section{Discussion}

The centrality analysis of PPIs usually leads to the introduction of a few key genes which play critical roles in network construction and also managing the altered biological processes under the studied condition. In the present study, 11 critical DEGs were identified in $S$. cerevisiae samples which were irradiated 30 s and harvested after 15 min. of laser radiation. Action map analysis results highlighted RPN11, UBI4, HSC82, and HSP82 as the crucial targets of laser radiation; however, UBI4 was determined as prominent DEGs. Based on the reported data, UBI4 was upregulated with a fold change of 3.9. As it is shown in Figure 2, RPN11 activated UBI4, while HSC82 and HSP82 inhibited UBI4. RPN11 was upregulated 2.24
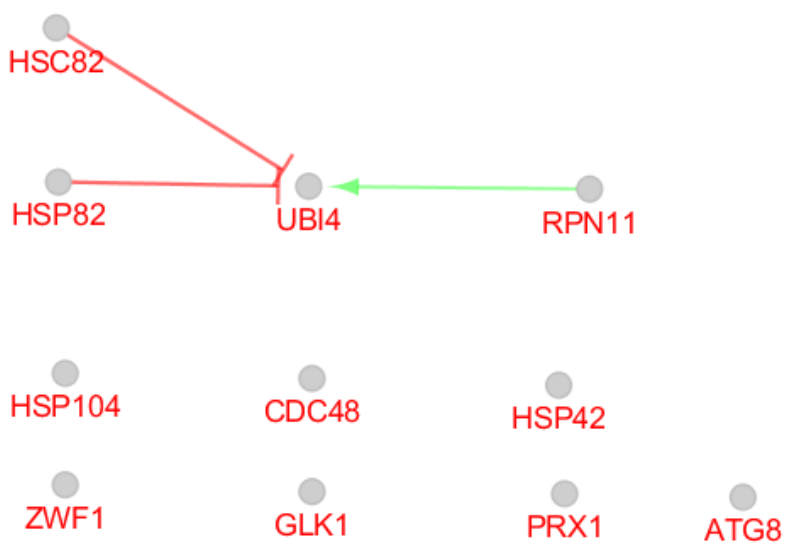

Figure 2. Action Map for the 11 Critical DEGs. Green and red arrows refer to activation and inhibition respectively.

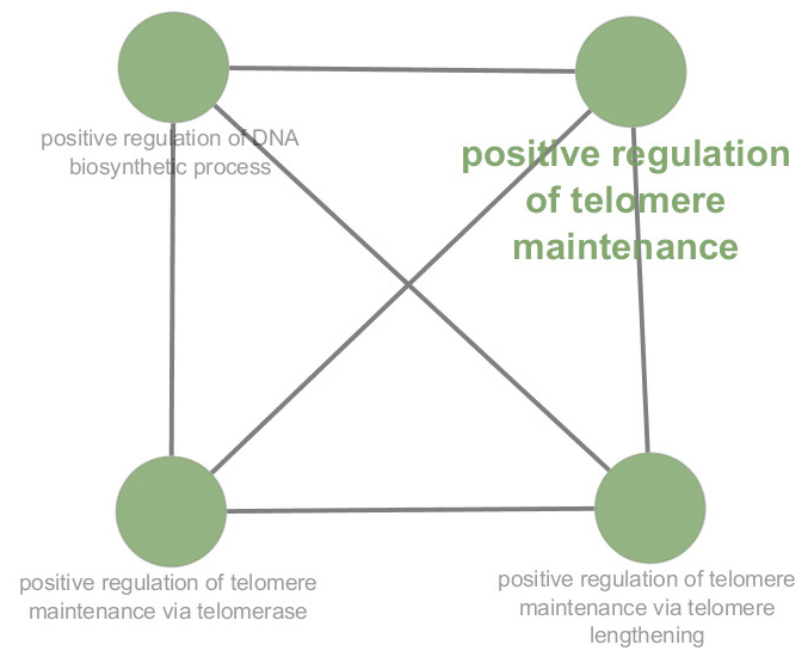

Figure 3. Biological terms Related to the Key Critical DEGs. The label of the term by which the class is characterized is colored. 
times versus HSC82 that was downregulated 2.3 times. HSP82, the inhibitor of UBI4, was upregulated about 7 times. It can be concluded that the overall upregulation and downregulation of activators and inhibitors lead to hyperactivation of UBI4 which is upregulated. This calculation is an estimation of the occurred processes. This finding indicates that upregulation and hyper-activation of UBI4 is the prominent advantage of laser radiation in the applied condition. Since "positive regulation of telomere maintenance" is the main biological term that is related to these 4 DEGs, it seems that telomere is an important target of the laser in the irradiated samples.

Telomeres are nucleoprotein structures on the chromosome end. Telomeres provide protection chromosome ends from enzymatic degradation and maintain chromosomal and genomic stability. Loss of telomere function may cause genetic instability and cancer progression. Chromosomal stability is the character of normal cells, while cancer cells have frequent chromosomal changes. ${ }^{27}$ Loss of a telomere results in chromosomal end-to-end fusion, chromosomal rearrangements and instability. ${ }^{28}$ The use of yeast as a simple model for the study of ultraviolet light on the structure of telomeres in this study showed a change in the expression of some genes in the genomic profile of yeast. Our results demonstrated that UBI4 expression was affected by RPN11 \& HSC82 and HSP82. UBI4 or polyubiquitin gene of $S$. cerevisiae is a modulator of life span, and UBI4 is induced in response to DNA damaging agents and heat shock. UBI4 is also expressed in higher levels in cells growing by respiration. ${ }^{29}$ UBI4 gene deficiency leads to early induction of apoptosis and shortened replicative lifespan in yeast. ${ }^{30}$ Zhao et al believe that the overexpression of UBI4 in yeast will increase resistance to toxin and the overexpression of UBI4 is associated with increased ubiquitin and ubiquitinated proteins. ${ }^{29}$ RPN11 could upregulate UBI4 according to our results. RPN11 is served as the essential deubiquitinase of the proteasome. On the other hand, HSC82 and HSP82 genes inhibit UBI4 based on our data. However, the overexpression of HSC82 and HSP82 resulted in telomere shortening in wild-type cells of yeast. ${ }^{31}$ Figure 3 demonstrates the relationship between our key genes and telomere maintenance with positive regulation according to ClueGO results. Our data suggest the connections between telomere survival and the effect of the UV laser on behalf of 4 key genes, namely RPN11, UBI4, HSC82, and HSP82, targeted as regulators.

\section{Conclusion}

The finding indicates that "positive regulation of telomere maintenance" is related to RPN11, UBI4, HSC82, and HSP82. This term is affected by 30s UV-laser radiation on the $S$. cerevisiae samples which are harvested $15 \mathrm{~min}$. after exposure. This result offers a new perspective on laser application in the rejuvenation process. It seems that laser potential in the promotion of life quality is higher than that of the usual aspects of its application in the present time of human life. It may be a suitable candidate against the aging process which is a limiting factor in human life. However, the possible side effects of laser application in human life should be investigated continuously.

\section{Acknowledgment}

Shahid Beheshti University of Medical Sciences supported this research.

\section{Conflicts of Interests}

The authors declare they have no conflicts of interest.

\section{Ethical Considerations}

Not applicable.

\section{References}

1. D'Orazio J, Jarrett S, Amaro-Ortiz A, Scott T. UV radiation and the skin. Int $J$ Mol Sci. 2013;14(6):12222-12248. doi:10.3390/ijms140612222

2. Armstrong BK, Kricker A. The epidemiology of UV induced skin cancer. J Photochem Photobiol B. 2001;63(13):8-18. doi: 10.1016/s1011-1344(01)00198-1.

3. Kaidzu S, Sugihara K, Sasaki M, Nishiaki A, Ohashi H, Igarashi $\mathrm{T}$, et al. Re-Evaluation of Rat Corneal Damage by Short-Wavelength UV Revealed Extremely Less Hazardous Property of Far-UV-C ${ }^{\dagger}$. Photochem Photobiol. 2021;97(3):505-516. doi: 10.1111/php.13419.

4. Basu AK. DNA Damage, Mutagenesis and Cancer. Int J Mol Sci. 2018 23;19(4):970. doi: 10.3390/ijms19040970.

5. de Laat A, van Tilburg M, van der Leun JC, van Vloten WA, de Gruijl FR. Cell cycle kinetics following UVA irradiation in comparison to UVB and UVC irradiation. Photochem Photobiol. 1996;63(4):492-7. doi: 10.1111/j.17511097.1996.tb03075.x.

6. Holick MF. Sunlight, UV-radiation, vitamin D and skin cancer: how much sunlight do we need? Adv Exp Med Biol. 2008;624:1-15. doi: 10.1007/978-0-387-77574-6_1.

7. Langton AK, Sherratt MJ, Griffiths CE, Watson RE. A new wrinkle on old skin: the role of elastic fibres in skin ageing. Int J Cosmet Sci. 2010;32(5):330-9. doi: 10.1111/j.14682494.2010.00574.x.

8. Liu-Smith F, Jia J, Zheng Y. UV-Induced Molecular Signaling Differences in Melanoma and Non-melanoma Skin Cancer. Adv Exp Med Biol. 2017;996:27-40. doi: 10.1007/978-3-319-56017-5_3.

9. Chen H, Weng QY, Fisher DE. UV signaling pathways within the skin. J Invest Dermatol. 2014;134(8):2080-2085. doi: 10.1038/jid.2014.161.

10. Suzuki I, Cone RD, Im S, Nordlund J, Abdel-Malek ZA. Binding of melanotropic hormones to the melanocortin receptor MC1R on human melanocytes stimulates proliferation and melanogenesis. Endocrinology. 1996;137(5):1627-1633. doi:10.1210/endo.137.5.8612494

11. Suzuki I, Cone RD, Im S, Nordlund J, Abdel-Malek ZA. Binding of melanotropic hormones to the melanocortin receptor MC1R on human melanocytes stimulates proliferation and melanogenesis. Endocrinology. 1996;137(5):1627-33. doi: 10.1210/endo.137.5.8612494.

12. Narayanan DL, Saladi RN, Fox JL. Ultraviolet radiation and skin cancer. Int J Dermatol. 2010;49(9):978-86. doi: 
10.1111/j.1365-4632.2010.04474.x.

13. Berwick M, Wiggins C. The current epidemiology of cutaneous malignant melanoma. Front Biosci. 2006;11:1244-54. doi: 10.2741/1877.

14. Ling G, Persson A, Berne B, Uhlén M, Lundeberg J, Ponten F. Persistent p53 mutations in single cells from normal human skin. Am J Pathol. 2001;159(4):1247-53. doi: 10.1016/S0002-9440(10)62511-4.

15. Hodis E, Watson IR, Kryukov GV, Arold ST, Imielinski $\mathrm{M}$, Theurillat J-P, et al. A landscape of driver mutations in melanoma. Cell. 2012;150(2):251-63. doi: 10.1016/j. cell.2012.06.024.

16. Rees JL. The genetics of sun sensitivity in humans. Am J Hum Genet. 2004;75(5):739-51. doi: 10.1086/425285.

17. Meyskens FL Jr, Farmer P, Fruehauf JP. Redox regulation in human melanocytes and melanoma. Pigment Cell Res. 2001;14(3):148-54. doi: 10.1034/j.16000749.2001.140303.x.

18. Schulz I, Mahler HC, Boiteux S, Epe B. Oxidative DNA base damage induced by singlet oxygen and photosensitization: recognition by repair endonucleases and mutagenicity. Mutat Res. 2000;461(2):145-56. doi: 10.1016/s09218777(00)00049-5.

19. Hoeijmakers JH. DNA damage, aging, and cancer. $N$ Engl J Med. 2009 8;361(15):1475-85. doi: 10.1056/ NEJMra0804615.

20. Nouspikel T. DNA repair in mammalian cells : Nucleotide excision repair: variations on versatility. Cell Mol Life Sci. 2009;66(6):994-1009. doi: 10.1007/s00018-009-8737-y.

21. Foury F. Human genetic diseases: a cross-talk between man and yeast. Gene. 1997;195(1):1-10. doi: 10.1016/s03781119(97)00140-6.

22. Schild D, Brake AJ, Kiefer MC, Young D, Barr PJ. Cloning of three human multifunctional de novo purine biosynthetic genes by functional complementation of yeast mutations. Proc Natl Acad Sci U S A. 1990;87(8):2916-20. doi: 10.1073/ pnas.87.8.2916.

23. Marx S, Vogelstein B, Kinzler K. Genetic Basis of Human Cancer. Eds B Vogelstein \& KW Kinzler New York: Mc Graw Hill. 1998 :489-506.

24. Said MR, Begley TJ, Oppenheim AV, Lauffenburger DA, Samson LD. Global network analysis of phenotypic effects: protein networks and toxicity modulation in Saccharomyces cerevisiae. Proceedings of the National Academy of Sciences (PNAS). 2004;101(52):18006-11. doi:10.1073/pnas.0405996101

25. Thomas S, Bonchev D. A survey of current software for network analysis in molecular biology. Hum Genomics. 2010;4(5):353-60. doi: 10.1186/1479-7364-4-5-353.

26. Hauser M, Abraham PE, Barcelona L, Becker JM. UV LaserInduced, Time-Resolved Transcriptome Responses of Saccharomyces cerevisiae. G3 (Bethesda). 2019;9(8):25492560. doi:10.1534/g3.119.400291

27. Lengauer C, Kinzler KW, Vogelstein B. Genetic instabilities in human cancers. Nature. 1998;396(6712):643-9. doi: $10.1038 / 25292$.

28. McClintock B. The Fusion of Broken Ends of Chromosomes Following Nuclear Fusion. Proc Natl Acad Sci U S A. 1942;28(11):458-63. doi: 10.1073/pnas.28.11.458.

29. Cheng L, Watt R, Piper PW. Polyubiquitin gene expression contributes to oxidative stress resistance in respiratory yeast (Saccharomyces cerevisiae). Mol Gen Genet. 1994;243(3):358-62. doi: 10.1007/BF00301072.

30. Zhao W, Zhou T, Zheng H-Z, Qiu K-P, Cui H-J, Yu H, et al. Yeast polyubiquitin gene UBI4 deficiency leads to early induction of apoptosis and shortened replicative lifespan. Cell Stress Chaperones. 2018;23(4):527-537. doi: 10.1007/ s12192-017-0860-3.

31. Grandin N, Charbonneau M. Hsp90 levels affect telomere length in yeast. Mol Genet Genomics. 2001;265(1):126-34. doi: $10.1007 / \mathrm{s} 004380000398$. 